\title{
molecules
}

ISSN 1420-3049

www.mdpi.com/journal/molecules

Article

\section{Halogen Bonding in (Z)-2-Iodocinnamaldehyde}

Pakorn Bovonsombat ${ }^{1, *}$, Francesco Caruso ${ }^{2,3}$, Andrew Jdaydani ${ }^{3}$ and Miriam Rossi ${ }^{3}$

1 Mahidol University International College, Mahidol University, Salaya Campus, Nakorn Pathom 73170, Thailand

2 Istituto Chimica Biomolecolare CNR, P.le Aldo Moro 5, Rome 00185, Italy

3 Department of Chemistry, Vassar College, Poughkeepsie, NY 12604-0484, USA

* Author to whom correspondence should be addressed; E-Mail: pakorn.bov@mahidol.ac.th.

Received: 24 May 2013; in revised form: 11 July 2013 / Accepted: 18 July 2013 /

Published: 24 July 2013

\begin{abstract}
Based on the bulkiness of the iodine atom, a non-planar conformation was expected for the title compound. Instead, its molecular structure is planar, as experimentally determined using single crystal X-ray diffraction, and confirmed theoretically by DFT calculations on the single molecule and the halogen pair paired molecules, therefore ruling out crystal packing forces as a principal factor leading to planarity. Indeed, planarity is ascribed to the carbonyl double bond, as when this bond is saturated on forming the related alcohol derivative, the molecule loses planarity. The X-ray molecular structure shows an intermolecular separation between the iodine and the oxygen of the carbonyl shorter than the corresponding van der Waals distance suggesting a weak halogen bond interaction. DFT minimization of this 2-molecule arrangement shows the iodine--oxygen distance much shorter than that observed in the crystal interaction and confirming its stronger halogen bond nature. A trend between increasing $\mathrm{I} \bullet \bullet \mathrm{O}$ (carbonyl) separation and decreasing $\mathrm{C}-\mathrm{I} \bullet \bullet \mathrm{O}$ (carbonyl) angle is demonstrated, further confirming the existence of a halogen bond.
\end{abstract}

Keywords: halogen bond; $\alpha$-iodoenals; crystal structure; DFT

\section{Introduction}

The iodo moiety in $\alpha$-iodo-enals and -enones is the preferred halogen in the asymmetric synthesis of several important biologically-active compounds such as (-)-brunsvigine [1], (-)-manthine [1], 
(-)-strychnine [2], shikimic acid analogues [3], fluoroneplanocin A [4] and (-)-tetrodotoxin [5]. Due to the ease, compared to other halogens, with which it undergoes carbon-carbon coupling, the iodine of $\alpha$-iodo-enals and -enones is also the preferred halogen substrate of choice in the Stille, Heck and Suzuki Pd-catalysed coupling reactions [6-10]. During the characterization of one of these aldehydes synthesized by our group, which involved a determination of the X-ray crystal structure of the title compound $[(Z)$-2-iodocinnamaldehyde], an intermolecular halogen interaction $\mathrm{C}-\mathrm{I} \bullet \bullet \mathrm{O}=\mathrm{C}$ was seen and is reported herein.

The definition of halogen bonds, shares many features with that of hydrogen bonds: they are attractive noncovalent interactions occurring when a covalently bound electron-deficient halogen atom pairs with nearby Lewis bases, in our case the carbonyl oxygen atom. Computational studies have shown that halogen bound organic donors produce weaker halogen bonds that are more electrostatic in nature [11]. Evidence for a weak electrostatic interaction between the positively polarized iodine atom and the nucleophilic carbonyl $\mathrm{O}$ atom is seen, experimentally, from the single crystal X-ray structure, and in calculations using DFT methods. The I $\cdots O \mathrm{O}$ distance is less than the sum of the van der Waals radii for iodine and oxygen and the $\mathrm{C}-\mathrm{I} \bullet \bullet \mathrm{O}$ angle $\left[149.33(5)^{\circ}\right]$ suggest a weak halogen bond in our molecule.

\section{Results and Discussion}

\subsection{Chemistry}

The direct synthesis of $\alpha$-iodoenones or $\alpha$-iodo- $\alpha, \beta$-unsaturated ketones from enones is well established [12-22]. However, examples of direct synthesis of linear $\alpha$-iodo- $\alpha, \beta$-unsaturated aldehydes ( $\alpha$-iodoenals) from enals are scarce and only two, those of (Z)-2-iodobutenal [23] and (Z)-2-iodocinnamaldehyde [24], are known thus far.

For the synthesis of (Z)-2-iodocinnamaldehyde (2), a published procedure for the synthesis of (Z)-2-bromocinnamaldehyde was adopted [24] (Scheme 1). $N$-iodosuccinimide (NIS) was employed as the source of iodine in the reaction, analogous to $N$-bromosuccinimide acting as the bromine donor in the synthesis of (Z)-2-bromocinnamaldehyde. In the iodination of trans-cinnamaldehyde (1), pyridine was more effective than pyridine- $N$-oxide and was utilised in the $n$-heptane-methanol solvent system, which consistently gave high yields of 2 ( $52 \%$ isolated yield) compared to those obtained from a single organic solvent system. The specificity for the $Z$-isomer is extremely high, with a $Z / E$ isomer ratio, as determined by GC-MS, of 405:1. Evidence for iodination at the $\alpha$-position of the enal comes from the absence of $\alpha$-hydrogen ${ }^{1} \mathrm{H}$-NMR absorptions in compound 2. Doublets, observed for the aldehyde hydrogens in 1 (caused by coupling of the $\alpha$-geminal vinyl hydrogen), were also absent in $\mathbf{2}$. These were instead replaced by singlets, which would be consistent with substitution of the $\alpha$-hydrogen by an iodine atom. The splitting pattern of the $\beta$-hydrogens of $\mathbf{2}$ was less complicated, becoming a triplet (due to the trans $\alpha$-hydrogen and the allyl hydrogen couplings). Nevertheless, iodo substitution at the $\alpha$-position can still furnish two geometric isomers, the $Z$-isomer 3, shown in an s-trans conformation, and the $E$-isomer $\mathbf{4}$. Furthermore, 3 can also exist in another conformation, the $s$-cis (5) (Figure 1). 
Scheme 1. Synthesis of $\mathbf{2}$ via direct $\alpha$-iodination of trans-cinnamaldehyde.

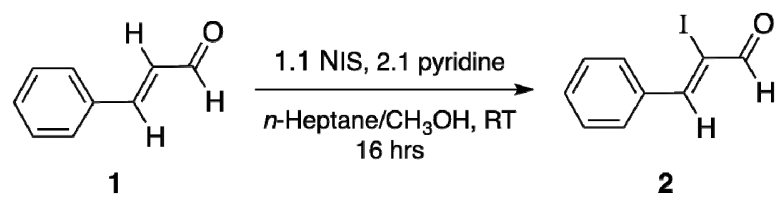

Figure 1. Isomers and conformations of 2.<smiles>O=C/C=C/c1ccccc1</smiles>

3<smiles>O=C/C(I)=C/c1ccccc1</smiles>

4<smiles>O=C/C(I)=C/c1ccccc1</smiles>

5

NOE experiments were performed to establish both the geometries and the conformations of 2. Irradiation of the $\beta$-hydrogen $\left(\mathrm{H}_{\beta}\right)$ of 2 gave a $6.9 \%$ intensity enhancement of the aldehyde hydrogen $\left(\mathrm{H}_{1}\right)$, and an $8.0 \%$ intensity enhancement for $\mathrm{H}_{\beta}$ was observed, when $\mathrm{H}_{1}$ was irradiated. The intensity enhancements of $\mathrm{H}_{1}$ and $\mathrm{H}_{\beta}$, when either was irradiated, are consistent with structure 3 thereby confirming the geometries of $\mathbf{2}$ as $Z$. In contrast, the geometric isomer $\mathbf{4}$ is not expected to show any intensity enhancements when either $\mathrm{H}_{1}$ or $\mathrm{H}_{\beta}$ is irradiated. Furthermore, the observed NOE effects firmly established that the conformation of $\mathbf{2}$ is s-trans, which would be consistent with $\mathbf{3}$ and not $\mathbf{5}$.

\subsection{Single Crystal X-ray Diffraction Study}

The X-ray structure of (Z)-2-Iodocinnamaldehyde shows the $s$-trans conformation of the enal structure, confirming the aforementioned COSY study and a wealth of information available on the conformation of enals (and enones) [25]. The almost planar molecular structure is depicted in Figure 2; the torsion angles $\mathrm{O}-\mathrm{C}-\mathrm{C}-\mathrm{I}\left[-3.9(2)^{\circ}\right]$ and $\mathrm{C}-\mathrm{C}-\mathrm{C}-\mathrm{I}\left[2.8(3)^{\circ}\right]$ are close to $0^{\circ}$, whereas the iodo-propenal fragment is out of the plane of the benzene ring, $\mathrm{C}-\mathrm{C}-\mathrm{C}-\mathrm{H}$ torsion angle $10.35(3)^{\circ}$ and similar to the unhalogenated cinnamaldehyde [26] C-C-C-H torsion angle 9.36(2) $)^{\circ}$ Also, we see a long $\mathrm{C}(\operatorname{aryl})-\mathrm{C}\left(\mathrm{sp}^{2}\right)$ $1.464(2) \AA$ similar to that seen in [26] (1.466(2) $\AA$ ) and indicating in both cases, poor resonance between the propenal fragment and the aromatic ring. The C-I bond length 2.085(2) $\AA$ is expected for a $\mathrm{C}\left(\mathrm{sp}^{2}\right)$-I, according to values seen in CSD. In [26] the carbonyl O atom has weak, 3.304(2) $\AA$, hydrogen bond with the para-H-C(aryl).

Figure 3 shows two out of eight molecules in the unit cell and an interaction between the iodine and the $\mathrm{O}$ (carbonyl) is seen. The I $\bullet \bullet \mathrm{O}$ separation of 3.418(2) $\AA$ is shorter than the sum of the $\mathrm{O}$ and I van der Waals radii of $3.50 \AA$, indicating a potential halogen bond. To exclude the possibility of this feature resulting from packing forces which could constrain the molecules to be closer than expected, a theoretical study was carried out.

\subsection{Theoretical Study}

It is known that in a $\mathrm{C}-\mathrm{X}$ bond, the halogen atom often acts as a Lewis acid since positive electrostatic potential is observed on it and directly opposite to the $\mathrm{C}$-X bond (the $\sigma$-hole). An example has been described for $\mathrm{CF}_{3} \mathrm{Br}$ [27]. 
Figure 2. X-ray molecular structure of the title compound including the two torsion angles, $-3.9^{\circ}$ and $2.8^{\circ}$ surrounding the iodine. These values, although small, are greater than the unsubstituted cinnamaldehyde [26] $\left(1.06^{\circ}\right.$ and $0.85^{\circ}$, respectively) due to the bulky iodine atom.

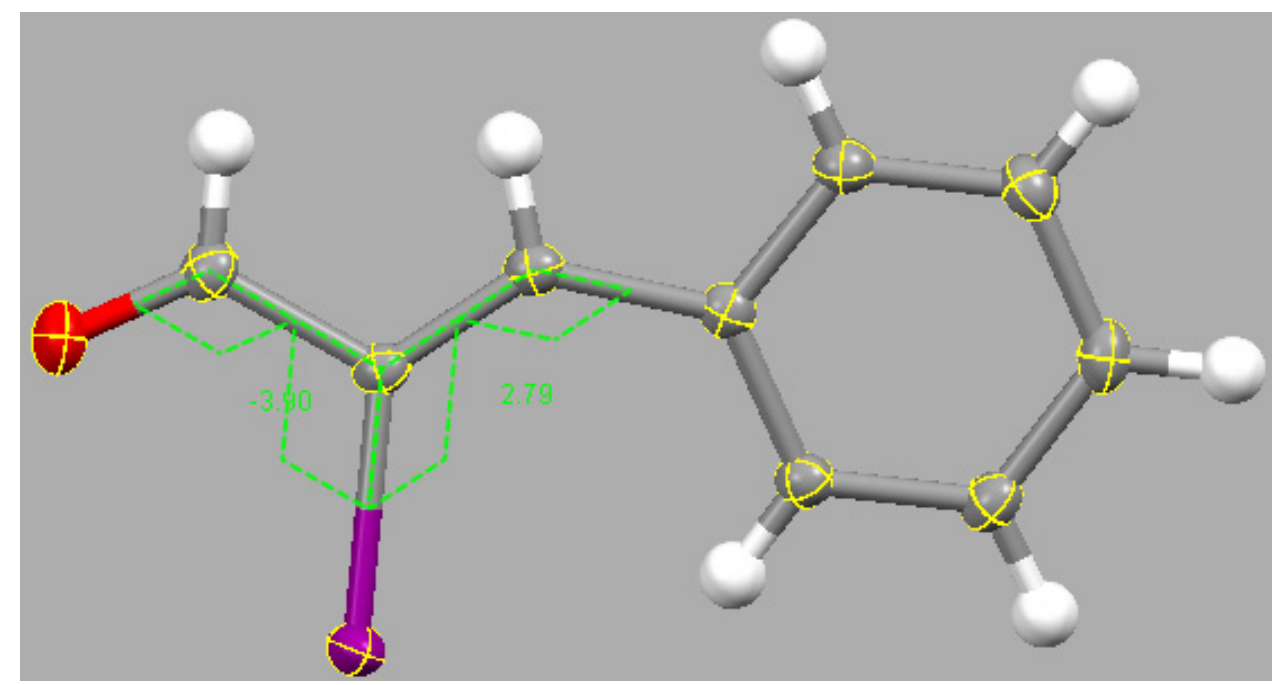

Figure 3. Intermolecular I--O separation (3.418 $\AA$ ) in the crystal, non-hydrogen atoms shown with their ellipsoids; C-I $\bullet \bullet O($ carbonyl $)=149.33(5)^{\circ}$.

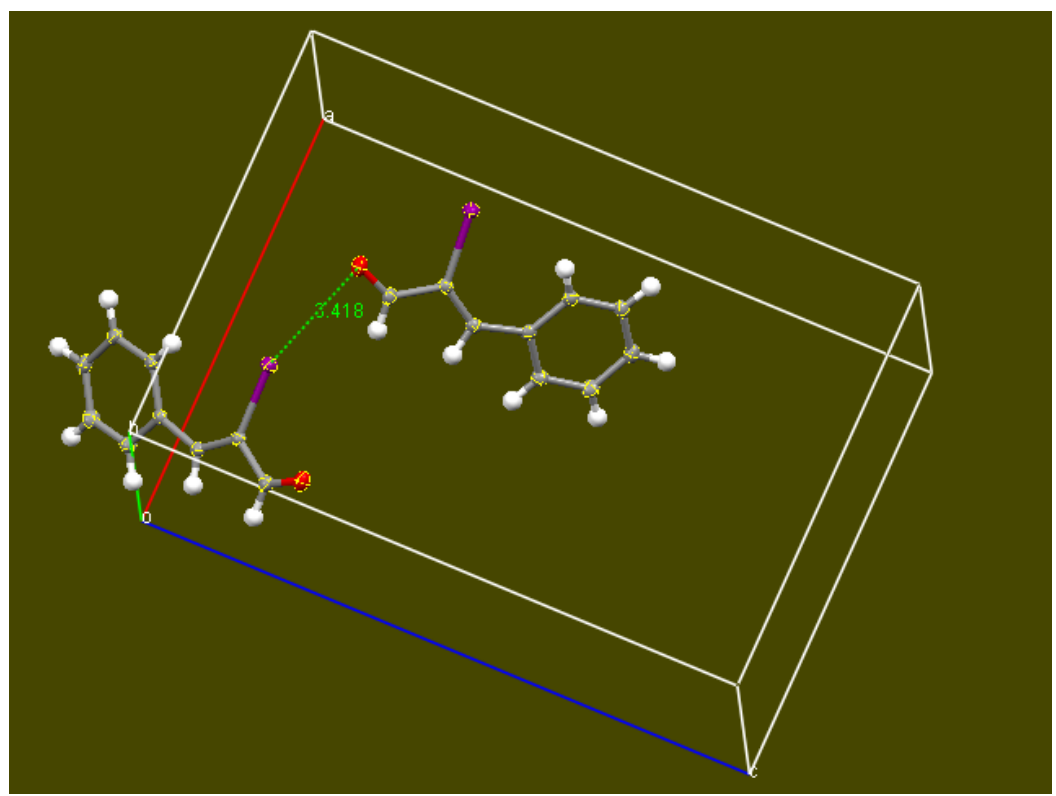

To verify the ability of $\mathrm{Dmol}^{3}$ to describe halogen bonds, we performed an ab-initio geometry optimization on a $\mathrm{CF}_{3} \mathrm{Br}$ molecule and obtained its electrostatic potential, as depicted in Figure 4. The area on the bromine atom but opposite to the $\mathrm{C}-\mathrm{Br}$ bond (left part) appears positively charged (red color), consistent with published results. 
Figure 4. $\mathrm{CF}_{3} \mathrm{Br}$ electrostatic potential, obtained after its geometry optimization, and using the same conditions for the title compound; the $\mathrm{Br}$ atom is located on the left, only two $\mathrm{F}$ atoms are visible on the right, and the $\mathrm{C}$ atom is seen as green shaded in the center. Color range blue-green-red (more negative (blue) ...green... less negative (red)).

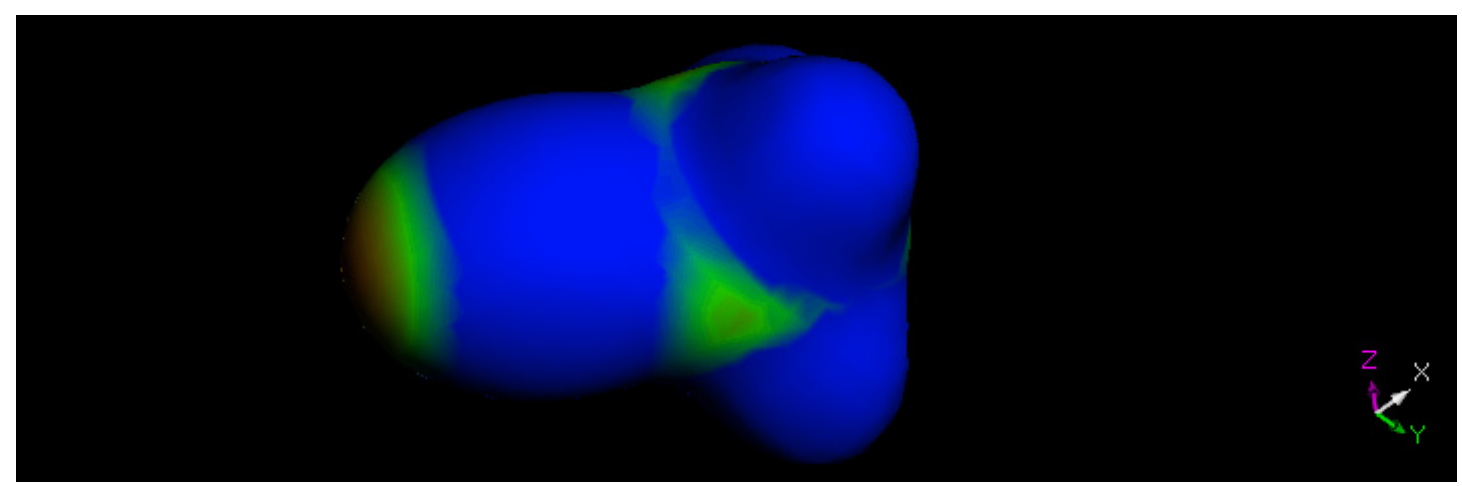

In our case, two molecules, as shown in Figure 3, were input in $\mathrm{Dmol}^{3}$ program and geometrically optimized. The converged system depicted in Figure 3 is at a minimum of energy. It is clear that an attractive interaction is present between both molecules; otherwise the geometry optimization would separate the molecules. In addition, this 2 -molecule calculated arrangement displays a $\mathrm{I} \bullet \bullet \mathrm{O}$ separation much shorter than in the crystal [3.083 $\AA$ vs. 3.418(2) $\AA$, respectively]. This suggests the packing in the crystal is not responsible for the short I $\bullet \bullet O$ intermolecular distance. Furthermore, halogen bonds display strong directionality according to Resnati and Metrangolo [28], and so we expect the O••I-C angle to be close to $180^{\circ}$, as confirmed in Figure 5.

Figure 5. After geometry optimization of the two-molecule arrangement in the crystal, the resulting DFT minimum arrangement is shown; green distance, yellow angle and light blue torsion angles.

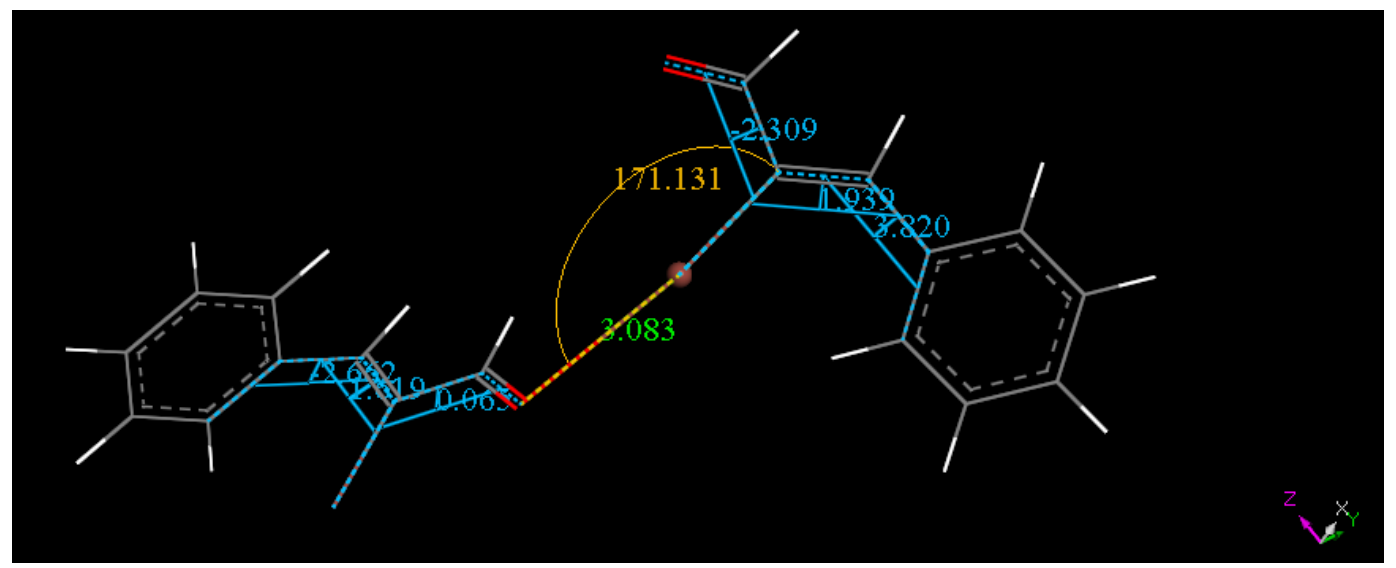

Comparing energies of two single molecules and the two-molecule arrangement of Figure 3, the latter is favored by $4.6 \mathrm{kcal} / \mathrm{mol}$. An additional calculation included a different two-molecule arrangement found in the crystal where I and $\mathrm{O}$ (carbonyl) were not related by intermolecular interactions; this arrangement was also more stable than two times the single molecule $(3.4 \mathrm{kcal} / \mathrm{mol})$ but not as much as the previous one. 
The single molecule, and the two-molecule arrangement (both as found in the crystal), were input in $\mathrm{Dmol}^{3}$ and the electrostatic potential calculated. Results are depicted in Figures 6 and 7 and appear consistent with earlier studies on halogen bonded compounds.

Figure 6. Electrostatic potential for the title compound in the crystal, the iodine atom is located in the upper right area and clearly shows and positive (red) $\sigma$-hole.

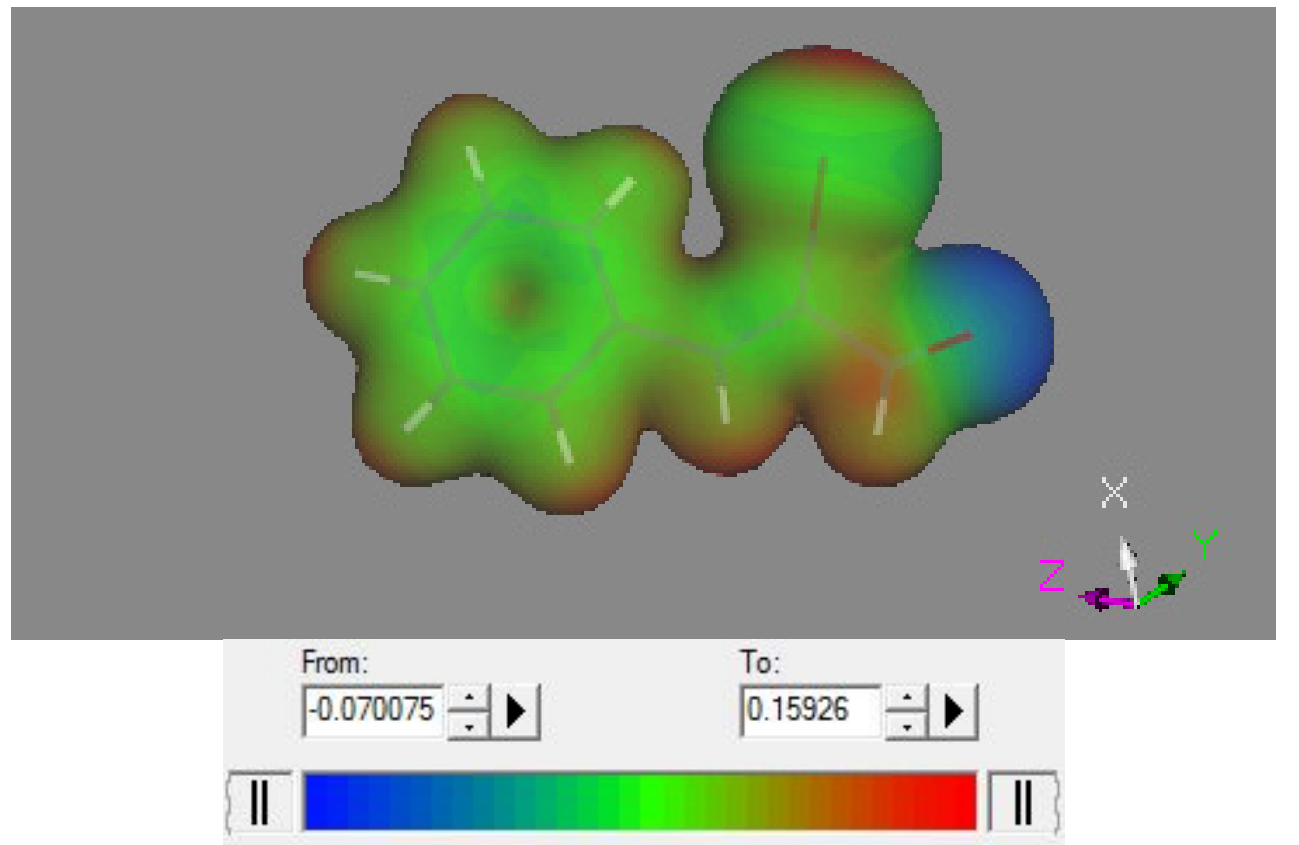

Figure 7. Electrostatic potential for the two-molecule arrangement in the crystal (also shown in Figure 3).

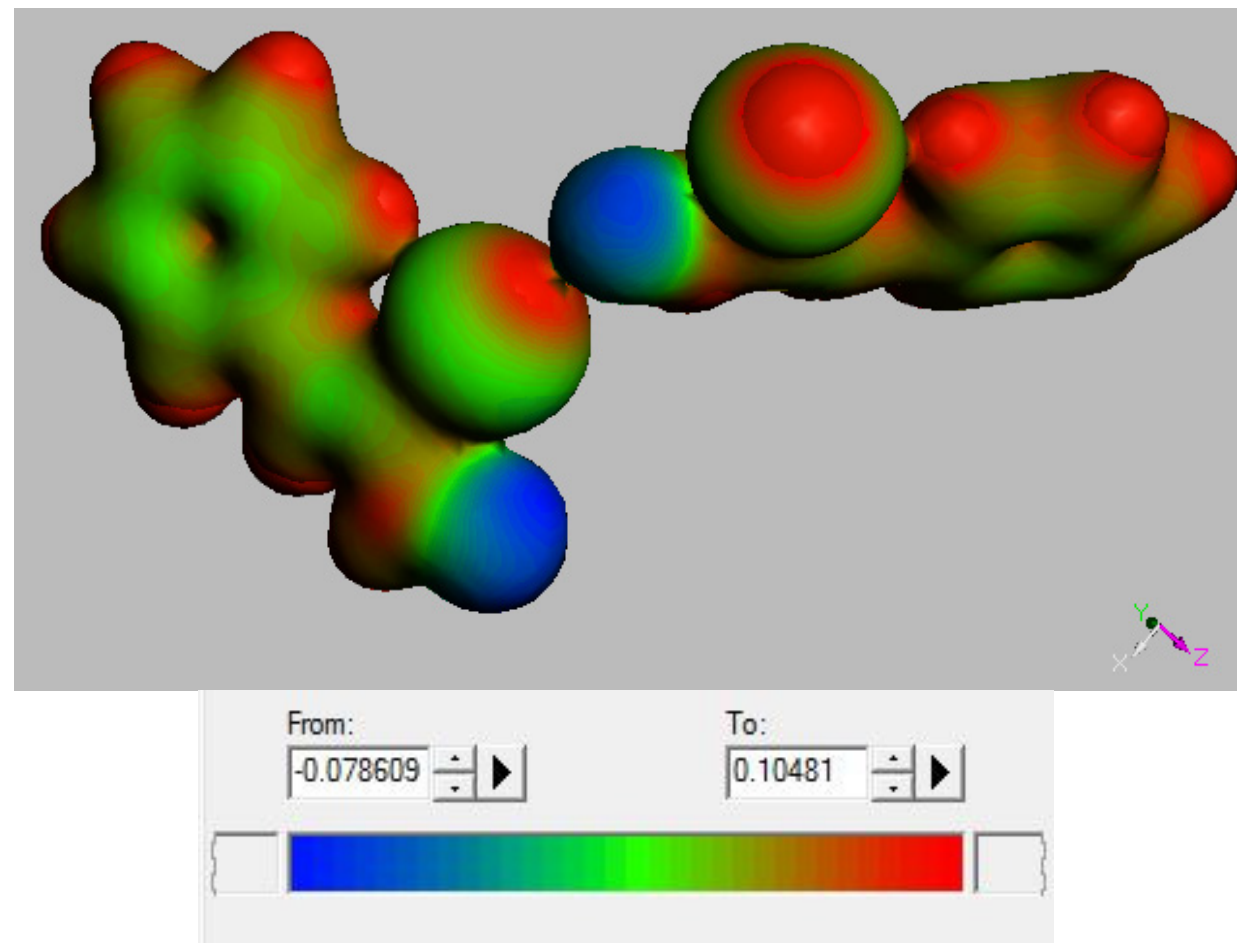


To further detect a halogen bond in the title compound we performed an additional calculation on the two-molecule arrangement. We selected and fixed several C-I $\bullet \cdot \mathrm{O}$ (carbonyl) angles and performed geometry optimization calculations. For a C-I $\cdots \bullet \mathrm{O}$ (carbonyl) angle of about $140^{\circ}$ the $\mathrm{I} \cdots \cdot \mathrm{O}$ (carbonyl) separation becomes $3.323 \AA$, which is longer than that of the real minimum $\left(171.1^{\circ}, 3.083 \AA\right)$. For a fixed C-I $\bullet \bullet \mathrm{O}$ (carbonyl) angle of about $120^{\circ}$, the $\mathrm{I} \bullet \bullet \mathrm{O}$ (carbonyl) separation $(3.595 \AA$ ) increases and is slightly longer than the van der Waals separation $(3.5 \AA)$, whereas a fixed C-I $\bullet \bullet O$ (carbonyl) angle of about $90^{\circ}$ results in even longer (3.862 $\AA$ ) $\mathrm{I} \bullet \bullet O$ (carbonyl) separation, suggesting both molecules drifting apart. Since halogen bonds, like hydrogen bonds, are more effective with angles about $180^{\circ}$ and absent for small angles, these results are consistent with halogen bond existence in the title compound.

In addition, in this series of calculation the smaller the angle the higher the energy, which indicates more stability for the non-fixed angle minimization $\left(\mathrm{C}-\mathrm{I} \bullet \bullet \mathrm{O}\right.$ (carbonyl) angle $=171.1^{\circ}$, Figure 5). Additional calculations were performed on the crystal, the cell was minimized using the same bases and functional. Variation in cell dimensions are found, $\mathrm{a}=11.262 \AA$ (11.473 $\AA$, Xray); $\mathrm{b}=8.849 \AA$

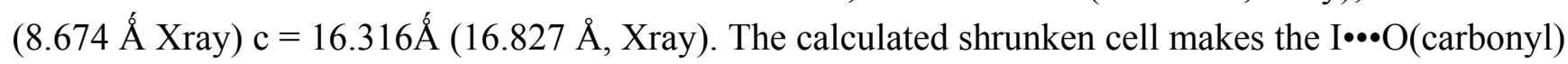
separation shorter $(3.347 \AA)$ than in the crystal, even shorter than van der Waals separation. We also calculated the lattice energy whose value is $-27.7 \mathrm{kcal} / \mathrm{mol}$.

As described above, the crystal structure of the title compound shows marked co-planarity in the molecule, in spite of the bulky iodine atom that might induce steric hindrance with the ortho-hydrogens of the phenyl ring. The question arises whether this co-planarity of the phenyl ring (with respect to the enal moiety) is due to both the carbonyl and the alkene, or is one of them sufficient to overcome the hindrance posed by the iodine? It seems obvious that saturating the alkene moiety should remove the co-planarity, but a carbonyl variation might disrupt the conjugation of the phenyl ring to the enal. In order to answer this question, a theoretical study on a variation of the carbonyl, the alcohol derivative, was undertaken. Figure 8 shows the co-planar DFT geometry optimized minimum carbonyl species, whereas the alcohol derivative optimized structure, Figure 9, loses co-planarity (torsion angle $=22.4^{\circ}$ ). Therefore, the effect of co-planarity is driven by the carbonyl moiety, which is an interesting example of long-range structural influence. The co-planarity finding is consistent with that described by Liljefors and Allinger [29], which showed enals possessing co-planarity conformation, even for those enals with methyl groups located geminal to the aldehyde or cis to the aldehyde. The calculations done by Liljefors and Allinger using molecular method (VESCF) predicted these enals to have an aldehyde-alkene dihedral angle of $180^{\circ}$. Furthermore the co-planar finding from the crystal structure of 2 is also consistent with the dipole study [30] and the findings from spectroscopic studies [31-34].

Figure 8. The geometrically optimized title compound.

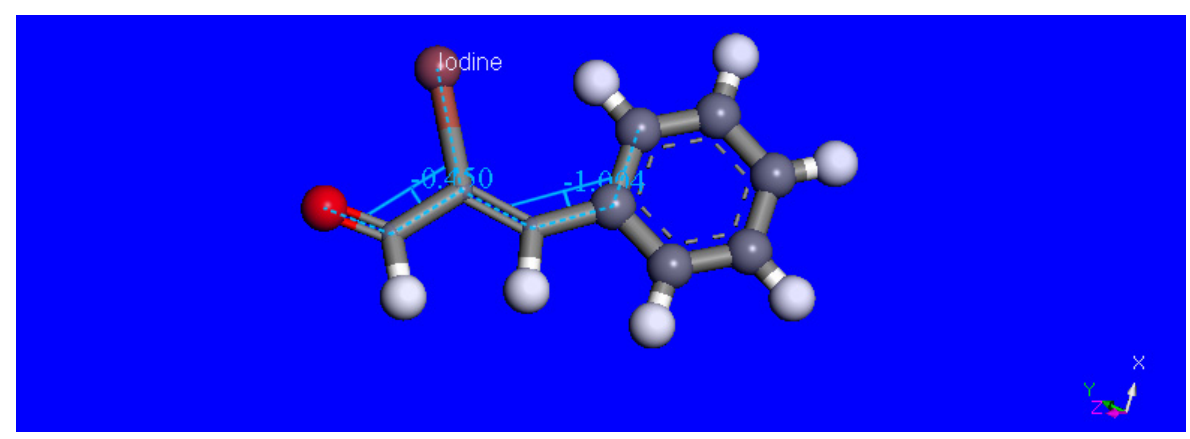


Figure 9. The geometrically optimized alcohol derivative, showing lack of co-planarity between the $\mathrm{Ph}$ ring and $\mathrm{C}=\mathrm{C}$ double bond (torsion angle $=22.45^{\circ}$ ).

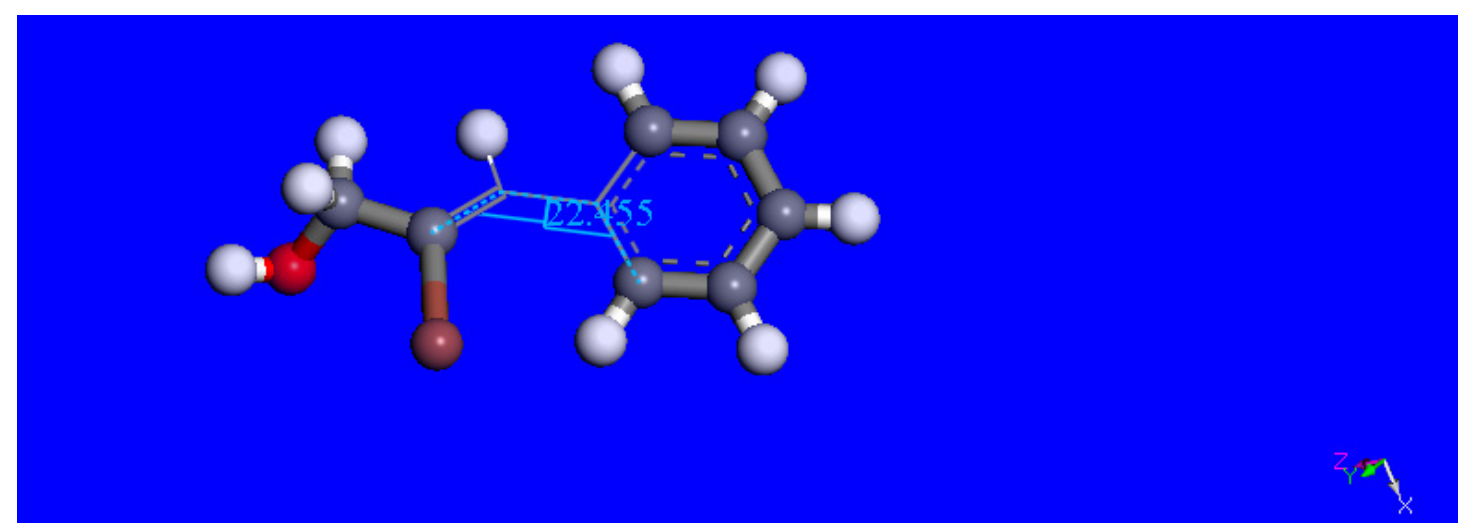

Our DFT optimized I--O interaction in the 2-molecule arrangement can be compared with that observed in the crystal structure [35] of methyl-2-chloro-5-iodo-4-(2-(1-(ethoxycarbonyl)ethylidene)hydrazino)benzoate $\left[\mathrm{d}(\mathrm{C}=\mathrm{O}--\mathrm{I})=3.073(8) \AA\right.$, $\left.\mathrm{O}--\mathrm{I}-\mathrm{C}=179.9(8)^{\circ}\right]$.

\section{Experimental}

\subsection{Chemistry}

trans-Cinnamaldehyde was obtained from Aldrich (St. Louis, MO, USA) and pyridine was from Fluka (Buchs, Switzerland). Acros Organics (Geel, Belgium) was the supplier of $N$-iodosuccinimide (NIS). The reagents were used without further purification. Methanol and n-heptane used in the reactions are of AR grade and were from RCI LabScan Co. Ltd. (Bangkok, Thailand). ${ }^{1} \mathrm{H}-$ and ${ }^{13} \mathrm{C}-\mathrm{NMR}$ spectra were recorded on a Bruker Avance $300 \mathrm{MHz}$ spectrometer in $\mathrm{CDCl}_{3}$ using TMS as an internal standard. The products composition and relative yields were carried out on a gas chromatograph-mass spectrometer (Agilent 6890 GC system and Agilent 5973 Mass Selective Detector) using HP-1 capillary column $(0.32 \mathrm{~mm} \times 24.9 \mathrm{~m} \times 017 \mu \mathrm{m})$. IR spectra were recorded on a Perkin-Elmer Spectrum 100 FT-IR Spectrometer. Separations of products were carried out on a centrifugal thin-layer chromatography (Harrison Research, Palo Alto, CA, USA) using a plate coated with $2 \mathrm{~mm}$ of silica gel $60 \mathrm{GF}_{254}$.

Synthesis Procedure of (Z)-2-iodo-3-phenylpropenal (2).

trans-Cinnamaldehyde $(63 \mu \mathrm{L}, 0.5 \mathrm{mmol})$ was dissolved in a methanol $(0.5 \mathrm{~mL})-n$-heptane $(9.5 \mathrm{~mL})$ solvent system, containing pyridine ( $85 \mu \mathrm{L}, 1.04 \mathrm{mmol}, 2.1$ equivs.). NIS (0.1284 g, $0.56 \mathrm{mmol})$ was then added in one portion to the solution and stirred at room temperature for 16 hours. The mixture was then diluted with $\mathrm{Et}_{2} \mathrm{O}(60 \mathrm{~mL})$ and washed with $10 \%$ sodium thiosulfate $(3 \times 20 \mathrm{~mL}), \mathrm{H}_{2} \mathrm{O}$ $(2 \times 20 \mathrm{~mL})$ and brine $(2 \times 10 \mathrm{~mL})$. The combined aqueous solution was re-extracted with $\mathrm{Et}_{2} \mathrm{O}$ $(10 \mathrm{~mL})$. The combined $\mathrm{Et}_{2} \mathrm{O}$ solution was dried over anhydrous $\mathrm{Na}_{2} \mathrm{SO}_{4}$. Purification of the iodo product was conducted with silica gel chromatography using $2 \% \mathrm{CH}_{2} \mathrm{Cl}_{2} /$ hexanes as the eluent. 
Compound 2: light yellow solid $\left(0.067 \mathrm{~g}, 52 \%\right.$ purified yield); $\mathrm{mp} 88-89{ }^{\circ} \mathrm{C}$ (lit. [24] mp $\left.89-90{ }^{\circ} \mathrm{C}\right)$; ${ }^{1} \mathrm{H}$ NMR (300 MHz, $\mathrm{CDCl}_{3}$ ): $\delta$ 7.44-7.57 (m, 3H, aromatic C-3, C-4 and C-5 Hs), 7.95-8.05 (m, 2H, aromatic C-2 and C-6 Hs), $8.10(\mathrm{~s}, 1 \mathrm{H}, \mathrm{CH}=\mathrm{CI}, \mathrm{NOE}$, enhancement from $\mathrm{CHO}, 7.9 \%), 8.79(\mathrm{~s}, 1 \mathrm{H}$, $\mathrm{CHO}$, NOE, enhancement from $\beta-\mathrm{H}, 6.9 \%) ;{ }^{13} \mathrm{C}-\mathrm{NMR}\left(75 \mathrm{MHz}, \mathrm{CDCl}_{3}\right): \delta 105.9,128.6,130.4,131.6$, 134.0, 155.8, 189.0; GC-MS (EI), $m / z$ (rel int.): $259\left(10,(\mathrm{M}+1)^{+}\right), 258\left(100, \mathrm{M}^{+}\right), 257\left(43,(\mathrm{M}-1)^{+}\right)$, $131\left(25,(\mathrm{M}-\mathrm{I})^{+}\right), 130\left(35,(\mathrm{M}-\mathrm{HI})^{+}\right), 127(5, \mathrm{I}), 103\left(76,((\mathrm{M}+1)-\mathrm{CHO})^{+}\right), 102\left(54,(\mathrm{M}-\mathrm{CHO})^{+}\right)$, $77\left(60, \mathrm{C}_{6} \mathrm{H}_{5}\right), 51(22)$; IR (ATR): 1669, $1612 \mathrm{~cm}^{-1}$.

\subsection{X-ray Diffraction Study}

Suitable crystals for X-ray diffraction of the title compound were obtained from an ether/hexane (3:7) solution at room temperature after about a week. Diffraction data were collected at $125 \mathrm{~K}$ using a Bruker SMART APEX II CCD X-ray diffractometer. Structure resolution and refinement were performed using SHELXTL [36]; details are included in Table 1. H atoms not found in Fourier maps were included from models and constrained as riding on their bound atoms. CCDC 940095 contains the supplementary crystallographic data for this paper. These data can be obtained free of charge via www.ccdc.cam.ac.uk/conts/retrieving.html (or from the CCDC, 12 Union Road, Cambridge CB2 1EZ, UK; fax: +44 1223 336033; e-mail: deposit@ccdc.cam.ac.uk).

Table 1. Crystal data and refinement details of (Z)-2-iodocinnamaldehyde.

\begin{tabular}{cc}
\hline Empirical formula & $\mathbf{C}_{9} \mathbf{H}_{7} \mathbf{I N O}$ \\
\hline Crystal color & colorless \\
Formula weight & 258.05 \\
Crystal System & Orthorhombic \\
Space group & P bca \\
Temperature $\mathrm{K}$ & $125(2)$ \\
Wavelength $(\AA)$ & 0.71073 \\
$a(\AA)$ & $11.4372(6)$ \\
$b(\AA)$ & $8.6736(5)$ \\
$c(\AA)$ & $16.8274(9)$ \\
Volume $\left(\AA^{3}\right)$ & $1669.31(16)$ \\
Z, density (mg/mm $\left.{ }^{3}\right)$ & $8,2.053$ \\
Absorption coefficient & 3.772 \\
Crystal size (mm) & $0.25 \times 0.17 \times 0.10$ \\
$\theta$ range data collection & $2.42,30.03$ \\
Limiting índices & $-16,16 /-12.12 /-23,23$ \\
Data collected /unique & 24123,2436 \\
Max, min. Transmission & $0.45 / 0.70$ \\
Refinement method & $\mathrm{F}^{2}$ \\
Refined data / parameters & $2057 / 128$ \\
Goodness-of-fit on $\mathrm{F}^{2}$ & 1.026 \\
Final R, Rw [I $>2$ sigma(I) & $0.0181 / 0.0408$ \\
\hline
\end{tabular}




\subsection{DFT Study}

Calculations were done using commercial software programs from Accelrys [37]. Density functional theory (DFT) code DMol3 was applied to calculate geometries, energies, and frequencies, implemented in Materials Studio 6.1, using a PC platform [38]. We employed Double Numerical Polarized (DNP) basis set that includes all the occupied atomic orbitals plus a second set of valence atomic orbitals plus polarized d-valence orbitals [39], and correlation generalized gradient approximation (GGA) was applied in the manner (PBE) suggested by Perdew-Burke-Ernzerhof [40]; the recent inclusion of a dispersion term that deals with $\mathrm{O} \cdot \bullet \mathrm{H}$ interactions was also applied (GRIMME) [41,42].

Spin unrestricted approach was exploited with all electrons being considered explicitly. The real space cutoff of $6 \AA$ was imposed for numerical integration of the Hamiltonian matrix elements. The self-consistent-field convergence criterion was set to the root-mean square change in the electronic density to be less than $10^{-6}$ electron $/ \AA^{3}$. The convergence criteria applied during geometry optimization were $2.7210^{-4} \mathrm{eV}$ for energy and $0.054 \mathrm{eV} / \AA$ for force.

\section{Conclusions}

Based on the bulkiness of the iodine atom and the potential steric hindrance posed on the ortho-hydrogens of the phenyl ring, the molecular structure of the title compound was expected to be non-planar. X-ray diffraction results demonstrated instead that the related torsion angles were close to $0^{\circ}$. Packing forces were not responsible for co-planarity as confirmed by DFT calculations, which also show molecular co-planarity. The driving force explaining co-planarity is assigned to the carbonyl double bond, as when it is saturated, forming the related alcohol derivative (replacing $\mathrm{HC}=\mathrm{O}$ by $\mathrm{H}_{2} \mathrm{COH}$ ), the molecule loses co-planarity. The X-ray molecular structure shows an intermolecular separation between the iodine and the oxygen of the carbonyl shorter than the corresponding van der Waals distance. DFT minimization of the molecular arrangement of the title compound makes the I $\cdots O$ interaction stronger as its distance is shorter than in the crystal, confirming its halogen bond nature.

\section{Supplementary Materials}

Supplementary materials can be accessed at: http://www.mdpi.com/1420-3049/18/8/8712/s1.

\section{Acknowledgements}

The financial support from Howard Hughes Medical Foundation, grant 52006322 to Vassar College. US National Science Foundation through grant 0521237 for the X-ray diffractometer. PB is sincerely grateful to Suchart Chaiyaroj and Chawannuch Ruaengsri of Ramathibodi Faculty of Medicine, Mahidol University for a life-saving operation.

\section{Conflict of Interest}

The authors declare no conflict of interest. 


\section{References and Notes}

1. Hong, A.-W.; Cheng, T.-H.; Raghukumar, V.; Sha, C.-K. An expedient route to Montanine-type Amaryllidaceae alkaloids: Total syntheses of (-)-brunsvigine and (-)-manthine. J. Org. Chem. 2008, 73, 7580-7585.

2. Ohshima, T.; Xu, Y.; Takita, R.; Shibasaki, M. Enantioselective total synthesis of (-)-strychnine: Development of a highly practical catalytic asymmetric carbon-carbon bond formation and domino cyclization. Tetrahedron 2004, 60, 9569-9588.

3. Begum, L.; Box, J.M.; Drew, M.G.B.; Harwood, L.M.; Humphreys, J.L.; Lowes, D.J.; Morris, G.A.; Redon, P.M.; Walker, F.M.; Whitehead, R.C. Difluorinated analogues of shikimic acid. Tetrahedron 2003, 59, 4827-4841.

4. Jeong, L.S.; Yoo, S.J.; Lee, K.M.; Koo, M.J.; Choi, W.J.; Kim, H.O.; Moon, H.R.; Lee, M.Y.; Park, J.G.; Lee, S.K.; et al. Design, Synthesis, and biological evaluation of fluoroneplanocin A as the novel mechanism-based inhibitor of S-adenosylhomocysteine hydrolase. J. Med. Chem. 2003, 46, 201-203.

5. Bamba, M.; Nishikawa, T.; Isobe, M. Stereoelectronic and steric control in chiral cyclohexane synthesis toward (-)-tetrodotoxin. Tetrahedron 1998, 54, 6639-6650.

6. Negishi, E. Novel and selective $\alpha$-substitution of ketones and other carbonyl compounds based on Pd-catalyzed cross coupling of $\alpha, \beta$-unsaturated carbonyl derivatives containing $\alpha$-halogen or $\alpha$-metal groups. J. Organomet. Chem. 1999, 576, 179-194.

7. Stille, J.K. The palladium-catalyzed cross-coupling reactions of organotin reagents with organic electrophiles. Angew. Chem. 1986, 25, 508-524.

8. Heck, R.F. Palladium reagents in organic synthesis. Org. React. 1982, 27, 345-390.

9. Suzuki, A.; Miyaura, N. Palladium-catalyzed cross-coupling reactions of organoboron compounds. Chem. Rev. 1995, 95, 2457-2483.

10. Scott, T.L.; Söderberg, B.C.G. Palladium-catalyzed synthesis of 1,2-dihydro-4(3H)-carbazolones. Formal total synthesis of murrayaquinone A. Tetrahedron 2003, 59, 6323-6332.

11. Chudzinski, M.G.; Taylor, M.S. Correlations between computation and experimental thermodynamics of halogen Bonding. J. Org. Chem. 2012, 77, 3483-3491.

12. McIntosh, J.M. Reaction of Iodine azide with cyclopentenone and cyclohexenone. Can J. Chem. 1971, 49, 3045-3047.

13. Jirkovsky, I. Studies on Enaminoketones. Can. J. Chem. 1974, 52, 55-65.

14. Smith, A.B.; Branca, S.J.; Guaciaro, M.A.; Wovkulich, P.M.; Korn, A. 2-Hydroxymethyl-2cyclopentenone. Org. Synth. Coll. Vol. VII 1990, 271-274.

15. Kim, T.H.; Asakura, J.; Asaka, Y. Reaction of enones with iodine in the presence of Ce(IV). Synthesis of 2-iodovinyl ketones. Chem. Express 1990, 5, 221-224.

16. Johnson, C.R.; Adams, J.P.; Braun, M.P.; Senanayake, C.B.; Wovkulich, P.M.; Uskokovic, M.R. Direct $\alpha$-iodination of cycloalkenones. Tetrahedron Lett. 1992, 33, 917-918.

17. Bovonsombat, P.; Angara, G.J.; McNelis, E. Concerning the formations of $\alpha$-iodoenones. Tetrahedron Lett. 1994, 35, 6787-6790.

18. Djuardi, E.; Bovonsombat, P.; McNelis, E. Formations of $\alpha$-Iodoenones by iodine and catalytic amounts of amines. Synth. Commun. 1997, 27, 2497-2503. 
19. Sha, C.K.; Huang, S.J. Synthesis of $\beta$-substituted $\alpha$-iodocycloalkenones. Tetrahedron Lett. 1995, 36, 6927-6928.

20. Roush, W.R.; Barda, D.A. Second generation synthesis of the quartromicin spirotetronic acid subunits via a Claisen rearrangement-intramolecular aldol sequence. Tetrahedron Lett. 1997, 38, 8785-8788.

21. Benhida, R.; Blanchard, P.; Fourrey, J.-L. A mild and effective iodination method using iodine in the presence of bis-(trifluoroacetoxy)iodobenzene. Tetrahedron Lett. 1998, 39, 6849-6852.

22. Krafft, M.E.; Cran, J.W. A convenient protocol for the $\alpha$-iodination of $\alpha, \beta$-unsaturated carbonyl compounds with $\mathrm{I}_{2}$ in an aqueous medium. Synlett 2005, 1263-1266.

23. Bowman, W.R.; Bridge, C.F.; Cloonan, M.O.; Leach, D.C. Synthesis of heteroarenes via radical cyclisation onto nitriles. Synlett 2001, 765-768.

24. Bovonsombat, P.; Rujiwarangkul, R.; Bowornkiengkai, T.; Leykajarakul, J. $\alpha$-Bromination of linear enals and cyclic enones. Tetrahedron Lett. 2007, 48, 8607-8610.

25. Patai, S.S., Rappoport, Z., Eds. The Chemistry of Enones, Part 1; Wiley: New York, NY, USA, 1989.

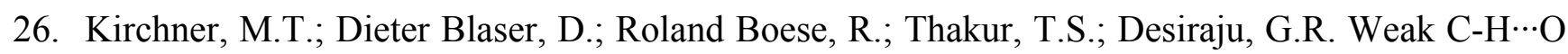
hydrogen bonds in anisaldehyde, Salicylaldehyde and cinnamaldehyde. Acta Cryst. 2011, C67, o387-o390.

27. Metrangolo, P.; Meyer, F.; Pilati, T.; Resnati, G.; Terraneo, G. Halogen bonding in supramolecular chemistry. Angew. Chem. Int. Ed. 2008, 47, 6114-6127.

28. Metrangolo, P.; Resnati, G., Eds. Halogen Bonding: Fundamentals and Applications, Structure and Bonding; Springer: Berlin, Germany, 2008; Volume 126.

29. Liljefors, T.; Allinger, N.L. Conformational analysis. CXII. Conformations, Energies, And electronic absorption spectra of $\alpha, \beta$-unsaturated aldehydes and ketones. J. Am. Chem. Soc. 1976, 98, 2745-2749.

30. Estok, G.K.; Dehn, J.S. Electric moments of some unsaturated carbonyl compounds. J. Am. Chem. Soc. 1955, 77, 4769-4770.

31. Suzuki, M.; Kozima, K. Microwave spectra, barrier heights to internal rotation of methyl group, and dipole moments for conjugated chain compounds: Methacrolein J. Mol. Spectrosc. 1971, 38, 314-321.

32. Hsu, S.L.; Flygare, W.H. Microwave spectrum and the barrier to internal rotation of the methyl group in trans- crotonaldehyde. Chem. Phys. Lett. 1969, 4, 317-319.

33. Forster, P.D.; Rao, V.M.; Curl, R.F., Jr. Microwave spectrum of Methyl Vinyl Ketone. J. Chem. Phys. 1965, 43, 1064-1066.

34. Forbes, W.F.; Shilton, R. Electronic spectra and molecular dimensions. III. ${ }^{1}$ Steric Effects in Methyl-substituted $\alpha, \beta$-Unsaturated Aldehydes. J. Am. Chem. Soc. 1959, 81, 786-790.

35. Chen, Y.; Shibata, M.; Rajeswaran, M.; Srikrishnan, T.; Dugar, S.; Pandey, R.K. Utility of Japp-Klingemann reaction for the preparation of 5-carboxy-6-chloroindole via Fischer indole protocol. Tetrahedron Lett. 2007, 48, 2353-2356.

36. Sheldrick, G.M. SHELXTL. PC version 5.1 An integrated system for solving, refining and displaying crystal structures from diffraction data, Bruker Analytical X-ray Systems, Karlsruhe, 2000.

37. Dmol3 Software; Accelrys, Inc.: San Diego, CA, USA, 2012. 
38. Delley, B. From molecules to solids with the DMol3 approach. J. Chem. Phys. 2000, 113, 7756-7764.

39. Delley, B. An all-electron numerical method for solving the local density functional for polyatomic molecules. J. Chem. Phys. 1990, 92, 508-517.

40. Perdew, J.P.; Burke, K.; Ernzerhof, M. Generalized gradient approximation made simple. Phys. Rev. Lett. 1996, 77, 3865-3868.

41. Grimme, S. Semiempirical GGA-type density functional constructed with a long-range dispersion correction. J. Comput. Chem. 2006, 27, 1787-1799.

42. Hanke, F.; Dyer, M.S.; Bjork J.; Persson, M. Structure and stability of weakly chemisorbed ethane adsorbed on low index $\mathrm{Cu}$ surfaces: Performance of density functionals with van der Waals interactions. J. Phys. Condensed Matter 2012, 42, 424217-424225.

Sample Availability: Samples of the compound (Z)-2-iodo-3-phenylpropenal (2) are available from the authors.

(C) 2013 by the authors; licensee MDPI, Basel, Switzerland. This article is an open access article distributed under the terms and conditions of the Creative Commons Attribution license (http://creativecommons.org/licenses/by/3.0/) 